\title{
S-P-1
}

\section{Acute Hypoxia Elevates Nitric Oxide Generation in Rat Carotid Body in Vitro}

Man-Lung Fung. ${ }^{1}$ Jian-Shan Ye. ${ }^{2}$ Peter Chin-Wan Fung. ${ }^{2}$

Departments of Physiology ${ }^{1}$ and Medicine ${ }^{2}$. Faculty of Medicine. The University of Hong Kong.

In acute hypoxia, the release of nitric oxide (NO) produced in rat carotid body is unclear. The concentration of $\mathrm{NO}$ was measured electrochemically with a Pt/Nafion/Pd-IrOx/POAP modified electrode placed on the surface of isolated carotid bodies superfused with bicarbonate-buffer saline at $35^{\circ} \mathrm{C}$. In hypoxia, the concentration of $\mathrm{NO}$ was increased by $17 \pm 2 \mathrm{nM}$ detected in a carotid body. The amount of NO release during hypoxia was augmented either by an increased number of carotid bodies surrounding the electrode or in the presence of L-arginine. In addition, the hypoxia-induced elevation of NO was abolished by the pretreatment of a nitric oxide synthase (NOS) inhibitor, $\mathrm{L}^{-\mathrm{N}^{\mathrm{G}}}$-nitroarginine methylester (L-NAME). Results suggest that endogenous NO production in the carotid body increases during hypoxia. Electrophysiological measurement of single fiber activity of the sinus nerve releaved that L-NAME treatment enhanced the afferent discharge in response to hypoxia. This confirms that the hypoxia-induced elevation of NO suppresses the carotid chemoreceptor response to hypoxia. Taken together, it was concluded that acute hypoxia increases the NO generation in the rat carotid body and the elevation of NO results in a suppression of carotid chemoreceptor activity during hypoxia. Hence, NO may play an active inhibitory role in the control of carotid chemoreceptor activity during hypoxa.

\section{S-P-2}

\section{Nitric Oxide Regulates Medium Potassium Channel of Cerebrocortical Neurons - A Patch- Clamp Study}

JS Ye, ${ }^{1}$ SC Zhang, ${ }^{2}$ FS Sheu, ${ }^{2}$ PCW Fung. ${ }^{1}$

${ }^{1}$ Division of Medical Physics, Department of Medicine, The University of Hong Kong, Hong Kong, China; ${ }^{2}$ Department of Biochemistry, University of Science and Technology, Clear Water Bay, Hong Kong, China.

Nitric Oxide (NO) can be generated endogenously by neuronal, endothelial, and inducible NO syntheses. It is of fundamental importance to understand the roles played by NO in the tissues and also at the cell level, if possible. As the cell functions are detrimentally influenced by ion channels, it is fruitful to use the patch clamp techniques for investigating the operational characteristics of the three ion channels in the presence of various concentrations of $\mathrm{NO}$ or its donor.

In this study, cerebroncortical neurons of newborn ICR mice were cultured. Special electrodes (of size $\sim 5$ microM) were made by us for single cell patch clamp study. The open and close time periods of medium-conductance $\sim 80 \mathrm{pohm}^{-1} \mathrm{~K}^{+}$channel were recorded and the open probability was calculated for each sample. The single neuron cell was subject to influence of SNAP (typical NO donor), SOD (superoxide dismuthase); strong reducing agent DTT and methylene blue were agents to analyze the plausible roles played by $\mathrm{NO}$ in regulating this $\mathrm{K}^{+}$channel. We have found that low concentration of SNAP ( 30 microM) would activate the opening process of this range of $\mathrm{K}^{+}$channel via a cGMPdependent pathway, but high concentration of SNAP ( 100 microM) would inhibit the channel instead by oxidizing the channel protein. Superoxide has similar inhibition effect as high concentration NO, also through oxidation of channel protein. For the past ten years or so, NO has demonstrated numerous protective effective as well as induced many cell/tissue toxicity in different models, under various conditions. For example, mycophenolate Mofetil (which inhibits inducible nitric oxide synthase generation during ischemia/reperfusion injury) has been considered as a potent immunosuppression drug while it is wellknown that NO-donor is a vasodilator. Understanding the fundamental roles (like regulation of ion channel) played by NO would provide key information on using NO donor, substrate, or NOS inhibitor as therapeutic measures of various diseases. 